OPEN ACCESS

Edited by:

Cyrille Delpierre,

Université Toulouse III Paul

Sabatier, France

Reviewed by:

Jose L. Flores-Guerrero,

University Medical Center

Groningen, Netherlands

Tony Kuo,

UCLA Fielding School of Public

Health, United States

*Correspondence:

Tonatiuh Barrientos-Gutiérrez

tbarrientos@insp.mx

Specialty section:

This article was submitted to Life-Course Epidemiology and Social Inequalities in Health,

a section of the journal

Frontiers in Public Health

Received: 05 August 2020 Accepted: 30 November 2020

Published: 28 January 2021

Citation:

Illescas-Zárate $D$, Batis $C$ Ramírez-Silva I, Torres-Álvarez $R$, Rivera JA and Barrientos-Gutiérrez $T$

(2021) Potential Impact of the

Nonessential Energy-Dense Foods

Tax on the Prevalence of Overweight and Obesity in Children: A Modeling Study. Front. Public Health 8:591696.

doi: 10.3389/fpubh.2020.591696

\section{Potential Impact of the Nonessential Energy-Dense Foods Tax on the Prevalence of Overweight and Obesity in Children: A Modeling Study}

Daniel Illescas-Zárate ${ }^{1}$, Carolina Batis ${ }^{2}$, Ivonne Ramírez-Silva ${ }^{1}$, Rossana Torres-Álvarez ${ }^{3}$, Juan A. Rivera ${ }^{1}$ and Tonatiuh Barrientos-Gutiérrez ${ }^{3 *}$

${ }^{1}$ Center for Nutrition and Health Research, National Institute of Public Health of Mexico, Cuernavaca, Morelos, Mexico, ${ }^{2}$ CONACYT-Center for Nutrition and Health Research, National Institute of Public Health, Cuernavaca, Morelos, Mexico,

${ }^{3}$ Center for Research in Population Health, National Institute of Public Health of Mexico, Cuernavaca, Morelos, Mexico

Background: Consumption of foods high in energy, sugar, fat, and salt contributes to the increase in body mass index and the prevalence of overweight and obesity in children. Mexico implemented an 8\% tax to non-essential energy-dense foods (NEDF) in 2014 as part of a national strategy to reduce obesity.

Objective: We modeled the potential effect of the NEDF tax on body mass index and overweight and obesity in Mexican children (6-17 years).

Materials and Methods: We used the Dynamic Childhood Growth and Obesity Model calibrated to Mexican children to simulate the potential 1-year effect of the NEDF tax on body weight. Inputs for the model included NEDF consumption, weight, and height, obtained from the 2012 Mexican National Health and Nutrition Survey. To project the potential impact of the tax, we ran a first simulation without intervention and another reducing the caloric intake from NEDF in the proportion observed in the Mexican population after the tax $(-5.1 \%)$. The tax effect was defined as the absolute difference in body mass index and prevalence of overweight and obesity between both models.

Results: The tax on NEDF should lead to a mean reduction of $4.1 \mathrm{~g}$ or $17.4 \mathrm{kcal} /$ day of NEDF at the population level. One year after the tax, mean body weight and body mass index should decrease $0.40 \mathrm{~kg}$ and $0.19 \mathrm{~kg} / \mathrm{m}^{2}$; this translates into -1.7 and $-0.4 \%$ points in overweight and obesity, respectively.

Conclusions: The use of fiscal instruments to discourage the consumption of NEDF could help to reduce the prevalence of overweight and obesity in children.

Keywords: overweight, obesity, energy balance, mathematical model, Mexico, children, taxed food 


\section{INTRODUCTION}

Childhood obesity is caused by poor regulation of energy balance over a long period of time, in which energy consumption is higher than a child's energy expenditure and normal body growth $(1-3)$. The prevalence of obesity in school-aged children and adolescents in Mexico is among the highest in the world (46). The rapid increase in childhood obesity is a major public health concern, leading to the development and implementation of structural interventions to improve diet $(7,8)$.

Consumption of non-essential energy-dense foods (NEDF) is considered to be a main driver of obesity (9). NEDF are high in added sugars, total fat, energy, and salt, can contribute to a significant proportion of the daily energy intake, and have been associated with weight gain $(10,11)$. To disincentivize their consumption, countries like Hungary, Denmark, and Australia recently established taxes to non-essential energy-dense foods (12).

In January 2014, Mexico passed an $8 \%$ tax to non-essential foods with an energy density exceeding $275 \mathrm{kcal} / 100 \mathrm{~g}$ (13); these foods contribute to 19.7 and $17.9 \%$ of the daily energy consumed by school-aged children and adolescents, respectively (14). One year after the implementation, a study estimated that the tax reduced NEDF household purchases by 5.1\% (15). However, to date, no study has analyzed the potential impact of this reduction in body weight in children.

We aimed to estimate the potential effect of the NEDF tax on body mass index (BMI) and the prevalence of overweight and obesity in Mexican children, using a mathematical model of childhood energy balance and body weight dynamics to project the expected weight change 1 year after the implementation of the NEDF tax.

\section{METHODS}

\section{Simulation Strategy}

Figure 1 summarizes the simulation strategy used. First, we obtained data of the pre-tax consumption of NEDF, age, sex, weight, and height from a representative sample of Mexican children and adolescents. Then, we calculated the change in NEDF consumption attributable to the tax for each child, assuming that observed changes in purchases translate into the same reductions in individual level consumption; expected changes in NEDF intake in grams were translated into calories. We then used a dynamic weight change model to estimate the 1-year weight change for each child assuming no intervention and an alternative model assuming the NEDF caloric reduction. The difference between scenarios is the projected change in body weight, body mass index, and overweight and obesity prevalence.

\section{Data Source}

Dietary and anthropometric information was obtained from the National Survey of Health and Nutrition 2012 (ENSANUT 2012, for its acronym in Spanish), which was conducted between

Abbreviations: NEDF, non-essential energy-dense foods; BMI, body mass index; ENSANUT 2012, Mexican National Health and Nutrition Survey 2012; 24HR, 24-h recall; SES, socioeconomic status; DCGO, Dynamic Childhood Growth and Obesity Model; FM, fat mass; FFM, free fat mass; CIs, confidence intervals.
October 2011 and May 2012 (16). ENSANUT is representative of the Mexican population at the national, regional, urban/rural, and state levels $(16,19)$. Trained interviewers conducted a 24 -h recall $(24 \mathrm{HR})$ in a random subsample $(\sim 11 \%$ of the ENSANUT respondents) and anthropometry in 10,886 subjects. Of this sample, we used information from two subgroups $(n=$ 4,140): school-aged children (6-11 years) and adolescents (12-17 years). Pregnant and/or lactating women were excluded from the analysis $(n=8)$.

\section{Anthropometry}

Body weight and height were obtained by trained and standardized personnel according to the procedures and protocols recommended for children $(20,21)$. We calculated BMI and excluded observations with implausible values $(<10$ or $>38 \mathrm{~kg} / \mathrm{m}^{2}$ for school-aged children and $<10$ or $>58 \mathrm{~kg} / \mathrm{m}^{2}$ for adolescents). BMI-for-age and height-for-age $z$-scores were estimated using the AnthroPlus software, as recommended by the World Health Organization (22). We considered plausible BMIfor-age $z$-scores between -5.0 and +5.0 standard deviations and between -6 or +6 standard deviations for height-for-age $z$-score. Four participants were excluded due to these criteria, as well as 153 who did not have anthropometric information.

The prevalence of overweight and obesity in school-aged children and adolescents were defined based on the World Health Organization criteria. Overweight was defined as BMI-for-age $z$-score $>1.0$ standard deviations and obesity as $>2.0$ standard deviations (18).

\section{Dietary Assessment}

Dietary information was collected using a $24 \mathrm{HR}$ proportionally distributed in the sample across all days of the week, including weekends (23). 24HR automated multiple-pass method was applied following the procedure recommended by the United States Department of Agriculture to minimize underreporting and improve the accuracy of recall (24). The mother or the person in charge of food preparation in the household was responsible for indicating the food consumed by children aged 15 or younger. Children older than 15 years were asked directly about their intakes. Dietary information was collected as (1) single foods such as the consumption of fruit, (2) custom recipes (characterized by the reported preparations where the study subject knew about the food and amounts used by participants), and (3) standard recipes (set of default ingredients that make up a recipe when the informer did not provide one). All recipes were disaggregated into their ingredients (except beverages) to facilitate identifying all NEDF in a recipe (e.g., chips, puffed wheat snacks, candies, chocolate, sweets, others) (23).

NEDF consumption was classified using the definition by the Ministry of Finance in conjunction with the Ministry of Health of Mexico. To be included in this classification, foods needed to comply with two conditions: an energy density $\geq 275 \mathrm{kcal} / 100 \mathrm{~g}$ and to not to be staple foods for the Mexican population, such as tortillas, toasts, whole grains, other cereals, and nonsweet bread. Food groups in this classification include French fries, salty snacks, cakes, chocolates, candies, sweet bread, frozen desserts, non-cereal based sweets, and ready-to-eat cereals (13). 


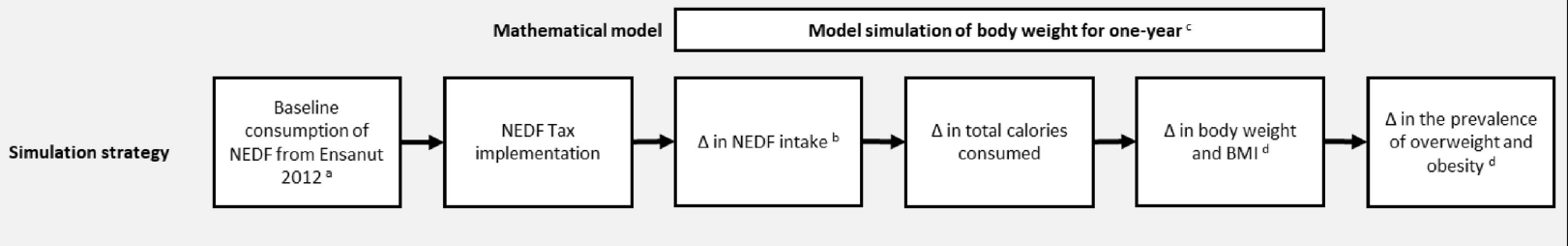

FIGURE 1 | Schematic illustration of the 1-year simulation strategy to estimate the potential effect of the NEDF tax on BMI and the prevalence of overweight and obesity in children aged 6-17 years. anputs for the model included Nonessential Energy-Dense Foods (NEDF) consumption, weight, and height, obtained from the

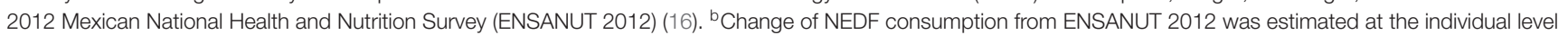
using an average of $5.1 \%$ reduction (15). ' Predicted body weight 1 year after the NEDF tax was simulated using the Dynamic Childhood Growth and Obesity model (DCGO) from Hall et al. (17). ${ }^{\mathrm{d} B o d y}$ mass index (BMI) and BMl-z-scores 1 year after the NEDF tax were obtained using the World Health Organization reference to estimate prevalences of overweight and obesity in children (18). We assume that all the change in body weight occurs via reduction in NEDF consumption.

Grams and energy of total consumption were calculated for each subject using the food composition base compiled by the National Institute of Public Health of Mexico (25).

We excluded 55 subjects with extreme values of energy consumption (exceeding \pm 3 standard deviations of the logarithm of the total energy requirement/total energy consumption ratio) and eight participants without diet information. Our analytical sample included 3,912 school-aged children and adolescents.

\section{Covariates}

Demographic variables such as sex, age, and socioeconomic status (SES) were obtained from ENSANUT 2012 (16). A socioeconomic status index was constructed using principal component analysis that included information on household characteristics and assets (26). The index was then divided using tertiles, to classify participants into low, medium, and high SES.

\section{Mathematical Model}

Dietary and anthropometric information from ENSANUT 2012 were used as inputs for the Dynamic Childhood Growth and Obesity Model (DCGO) proposed and validated by Hall et al. (17). Briefly, the DCGO model is a dynamic weight change model that uses a system of differential equations to predict changes in body weight over time, as energy intake or physical activity vary. An overview of the model and its calibration to the Mexican population by nutritional status is presented in the Supplementary Material. The DCGO model was designed to differentiate healthy growth between male and female individuals, considering the increase in energy consumption as age progresses and the physiological processes that stimulate the synthesis of fat free mass during childhood. As part of energy expenditure, it is assumed that physical activity remains constant from 6 to 12 years old; thereafter, physical activity decreases progressively as age increases (17).

\section{Change in NEDF Consumption Due to the Tax}

To estimate the reduction in consumption of NEDF, which is used as an input for the DCGO model, three studies were used as a reference. These studies evaluated the mean decrease in NEDF purchases in the first 3 years after the tax was implemented (2014, 2015 , or 2016), which estimated a $5.1 \%$ reduction in purchases of
NEDF for the first year (2014 vs. 2013 and 2012) (15) and 6.0\% for the first 2 years (2014 and 2015 vs. 2012) (27). The third study was an evaluation performed using the National Survey of Household Income and Expenditure of Mexico in 2014 and 2016, where a mean reduction of $5.3 \%$ in NEDF purchase was observed (28).

To maintain a conservative scenario, we assumed that the $5.1 \%$ decrease in purchases observed in the first year of the tax translated into the same reduction in NEDF consumption at the individual level. To capture differences by SES, we used the effects stratified as provided in the manuscript: 10.2, 5.8, and $0 \%$ for low, medium, and high SES, respectively (15). To translate this reduction into our sample, we first calculated the total grams of NEDF consumption as reported for each child; then, we multiplied it by the percent reduction that corresponded to his/her SES. Subsequently, the change in grams of NEDF was transformed into calories, assuming a mean NEDF energy density of $430 \mathrm{kcal} / 100 \mathrm{~g}$, as previously estimated (15). Thus, for instance, for a low-SES child who consumed $30 \mathrm{~g}$ of NEDF at baseline, the tax is expected to decrease $3.06 \mathrm{~g}$ of NEDF, equivalent to $13.16 \mathrm{kcal}$.

\section{Model Specification}

The DCGO model was used to obtain two scenarios of body weight in the sample with a 1-year simulation time frame. The first scenario assumed no intervention. The second scenario was simulated using the same inputs as scenario 1 but under the effect of the NEDF tax. To estimate BMI at the end of the modeling year, we used the body weight estimated by the model and projected the growth in height assuming that each child maintained the same height-for-age $z$-score. The prevalence of overweight and obesity were estimated using the BMI-for-age $z$-score. The expected impact of the NEDF tax was defined as the absolute difference between the model under the tax and the model without intervention 1 year after implementation. This estimate assumes that calories reduced by the NEDF tax are not compensated by other foods; as a sensitivity analysis, we used 10 and $20 \%$ energy compensation.

\section{Statistical Analysis}

We estimated calories per capita from NEDF and the percentage of consumption that these foods contribute to total energy consumption. In addition, reduction in grams and energy of 
NEDF attributed to tax implementation was estimated. These variables were described with mean and standard error according to age, sex, and SES.

The expected impact of NEDF tax in body weight, BMI, BMIfor-age $z$-score, and in the prevalence of overweight and obesity was calculated at the national level, by groups of age, sex, and SES. Body weight, BMI, and BMI-for-age $z$-score were described with means and standard error and prevalence as percent and $95 \%$ confidence intervals. The DCGO model does not produce confidence intervals (CIs) to the estimators; hence, our CIs were constructed using the error captured by the survey data.

Data processing of demographic, anthropometric, and dietary information, as well as analyses considering the survey design and sampling weights were performed using Stata, version 13 (Stata Statistical Software; StataCorp) (29). The DCGO model was implemented using the Dynamic Body Weight Model Package for Children and Adults in R software (30).

\section{RESULTS}

Table 1 shows the characteristics of selected children from ENSANUT 2012. We included 3,912 participants, representing 27.9 million children between 5 and 17 years old in Mexico. The mean consumption of NEDF was $355 \mathrm{kcal} /$ day/capita or $17.7 \%$ of total energy consumption. The expected NEDF reduction in consumption as a result of the tax would translate into a mean decrease of $4.1 \mathrm{~g}$ or $17.4 \mathrm{kcal}$ per child per day. Table 2 shows

TABLE 1 | Characteristics of children 6-17 years of age in Mexico; 2012 Mexican National Survey of Health and Nutrition (ENSANUT 2012).

\begin{tabular}{|c|c|c|c|}
\hline & \multicolumn{3}{|c|}{ Total sample } \\
\hline & $n$ & $N^{\star}$ & Percentage/mean (SE) \\
\hline \multicolumn{4}{|l|}{ SEX (\%) } \\
\hline Male & 1,974 & 14,025 & 50.2 \\
\hline Female & 1,938 & 13,890 & 49.7 \\
\hline \multicolumn{4}{|l|}{ AGE } \\
\hline Mean (SE), years & 3,912 & 27,916 & $10.8 \pm 0.07$ \\
\hline School-aged children (\%) & 2,590 & 17,389 & 62.4 \\
\hline Adolescent (\%) & 1,322 & 10,527 & 37.6 \\
\hline \multicolumn{4}{|l|}{ AREA (\%) } \\
\hline Urban & 2,386 & 19,407 & 69.5 \\
\hline Rural & 1,526 & 8,508 & 30.4 \\
\hline \multicolumn{4}{|c|}{ SOCIOECONOMIC STATUS (\%) } \\
\hline Low & 1,452 & 9,288 & 33.2 \\
\hline Medium & 1,376 & 9,533 & 34.1 \\
\hline High & 1,084 & 9,094 & 32.5 \\
\hline Weight (kg) & 3,912 & 27,916 & $39.4 \pm 0.40$ \\
\hline Height (cm) & & & $139.2 \pm 0.43$ \\
\hline BMI $\left(\mathrm{kg} / \mathrm{m}^{2}\right)$ & & & $19.4 \pm 0.11$ \\
\hline BMI for Age, z-score & & & $0.58 \pm 0.03$ \\
\hline Height for Age, z-score & & & $-0.48 \pm 0.02$ \\
\hline
\end{tabular}

Results are presented in percentages or mean \pm standard error. *In thousands.

BMI, body mass index. that reductions in NEDF calories after the tax should be larger in low and medium SES than in high SES (28.1, 21.3 vs. $0 \mathrm{kcal}$, respectively), in adolescents than in school-aged children (18.5 vs. $16.7 \mathrm{kcal})$, and in male than in female children (18.0 vs. 16.8 $\mathrm{kcal})$.

Figure 2 shows the difference in body weight at the end of the simulation year, by sociodemographic characteristics. The tax is expected to reduce $-0.40 \mathrm{~kg}$ of body weight and $-0.19 \mathrm{~kg} / \mathrm{m}^{2}$ of BMI. Children with low SES $(-0.64 \mathrm{~kg})$, adolescents $(-0.46 \mathrm{~kg})$, and male children $(-0.41 \mathrm{~kg})$ are expected to experience the largest reductions in body weight.

Table 3 shows the expected changes in the prevalence of overweight and obesity in Mexican children after the implementation of the NEDF tax. One year after the tax, the prevalence of overweight should decrease -1.7 pp (95\% CI: $-0.8,-2.5)$, while obesity should decrease -0.4 pp (95\% CI: $-0.1,-0.6)$. Larger reductions in the prevalence of overweight should occur in school-aged children [ -1.8 pp (95\% CI: -0.8 , $-2.7)]$, male children [ -1.9 pp (95\% CI: $-0.5,-3.1)]$, and low SES children [-1.9 pp $(95 \% \mathrm{CI}:-0.80,-2.9)]$. However, the largest impact of the tax should be experienced by children in the medium SES group, with a $2.6 \mathrm{pp}$ reduction in overweight (95\% CI: $-0.60,-4.6)$. Reductions in the prevalence of obesity should be larger in school-aged children [-0.4 pp (95\% CI: $-0.1,-0.8)]$, male children $[-0.5$ pp $(95 \%$ CI: $-0.1,-0.9)]$, and in children in the low SES group [-0.6 pp (95\% CI: $-0.01,-1.0)]$. Point estimates and $95 \%$ confidence intervals for expected changes in BMI and BMI-for-age $z$-score are available in Supplementary Table 1.

Table 4 shows 1-year-simulation results of the sensitivity analysis, testing the potential effect of tax on NEDF assuming a 10 and $20 \%$ energy compensation. In these models, we found a slight attenuation of the expected impact in BMI, BMI-for-age $z$ score, and overweight prevalence but a similar reduction in the prevalence of obesity than without dietary compensation.

\section{DISCUSSION}

We modeled the potential effect of the $8 \%$ NEDF tax in Mexico on body weight and the prevalence of overweight and obesity in children between 5 and 17 years old. According to our estimates, the observed reductions in NEDF consumption after the tax should result in a national average reduction in energy consumption of $17.4 \mathrm{kcal} /$ person/day and a weight difference of $-0.40 \mathrm{~kg},-0.39 \mathrm{~kg} / \mathrm{m}^{2}$ in $\mathrm{BMI}$, and $\mathrm{a}-1.7$ and $\mathrm{a}-0.4 \mathrm{pp}$ in the prevalence of overweight and obesity at the end of the first year with the tax. Children in the low and middle SES groups will benefit from the tax, although the middle group will experience a larger difference. Children in the high SES group are not expected to benefit from this policy, given that no reduction in NEDF consumption has been observed in this group (15).

NEDF consumption is an important source of energy in Mexican children that has been proven to be reduced with the NEDF tax. NEDF are large contributors to the total energy consumption in Mexican children between 5 and 19 years old, with an estimated $18.8 \%$ of the daily caloric intake (14). An 
TABLE 2 | Non-essential energy-dense foods consumption and estimated impact of tax according to demographic characteristics in Mexican children aged 6-17 years according to ENSANUT 2012.

\begin{tabular}{|c|c|c|c|c|c|c|c|}
\hline & \multicolumn{2}{|c|}{ Sample } & \multirow{2}{*}{$\begin{array}{l}\text { Total energy consumption }{ }^{a} \\
\text { Calories per capita }\end{array}$} & \multicolumn{2}{|c|}{ NEDF consumption ${ }^{a}$} & \multicolumn{2}{|c|}{ Mean reduction due NEDF tax } \\
\hline & $n$ & $N^{*}$ & & Calories per capita & $\%$ of total calories & Grams $^{a}$ & Calories $^{b}$ \\
\hline Total & 3,912 & 27,916 & $1,927 \pm 21$ & $355 \pm 10$ & $17.7 \pm 0.4$ & $4.1 \pm 0.1$ & $17.4 \pm 0.5$ \\
\hline \multicolumn{8}{|l|}{ AGE GROUPS } \\
\hline School-aged children & 2,590 & 17,389 & $1,834 \pm 24$ & $341 \pm 11$ & $17.5 \pm 0.4$ & $3.9 \pm 0.1$ & $16.7 \pm 0.5$ \\
\hline Adolescent & 1,322 & 10,527 & $2,079 \pm 34$ & $378 \pm 15$ & $17.9 \pm 0.7$ & $4.3 \pm 0.2$ & $18.5 \pm 0.8$ \\
\hline \multicolumn{8}{|l|}{ SEX } \\
\hline Male & 1,974 & 14,025 & $2,032 \pm 31$ & $368 \pm 12$ & $17.3 \pm 0.6$ & $4.2 \pm 0.2$ & $18.0 \pm 0.6$ \\
\hline Female & 1,938 & 13,890 & $1,820 \pm 25$ & $342 \pm 13$ & $17.9 \pm 0.6$ & $3.9 \pm 0.2$ & $16.8 \pm 0.6$ \\
\hline \multicolumn{8}{|c|}{ SOCIOECONOMIC STATUS } \\
\hline Low & 1,452 & 9,288 & $1,882 \pm 34$ & $285 \pm 12$ & $15.1 \pm 0.6$ & $6.5 \pm 0.2$ & $28.1 \pm 0.6$ \\
\hline Medium & 1,376 & 9,533 & $1,960 \pm 34$ & $383 \pm 16$ & $18.6 \pm 0.7$ & $4.9 \pm 0.2$ & $21.3 \pm 0.7$ \\
\hline High & 1,084 & 9,094 & $1,936 \pm 37$ & $397 \pm 19$ & $19.2 \pm 0.8$ & 0 & 0 \\
\hline
\end{tabular}

Results are presented in mean \pm standard error.

*In thousands.

aInformation about consumption and demographic characteristics of this table were obtained from the 2012 Mexican National Health and Nutrition Survey (ENSANUT 2012) (16).

${ }^{b}$ Calories estimated from assuming a mean energy density of the NEDF of $430 \mathrm{kcal} / 100 \mathrm{~g}$.

NEDF, non-essential energy-dense foods.

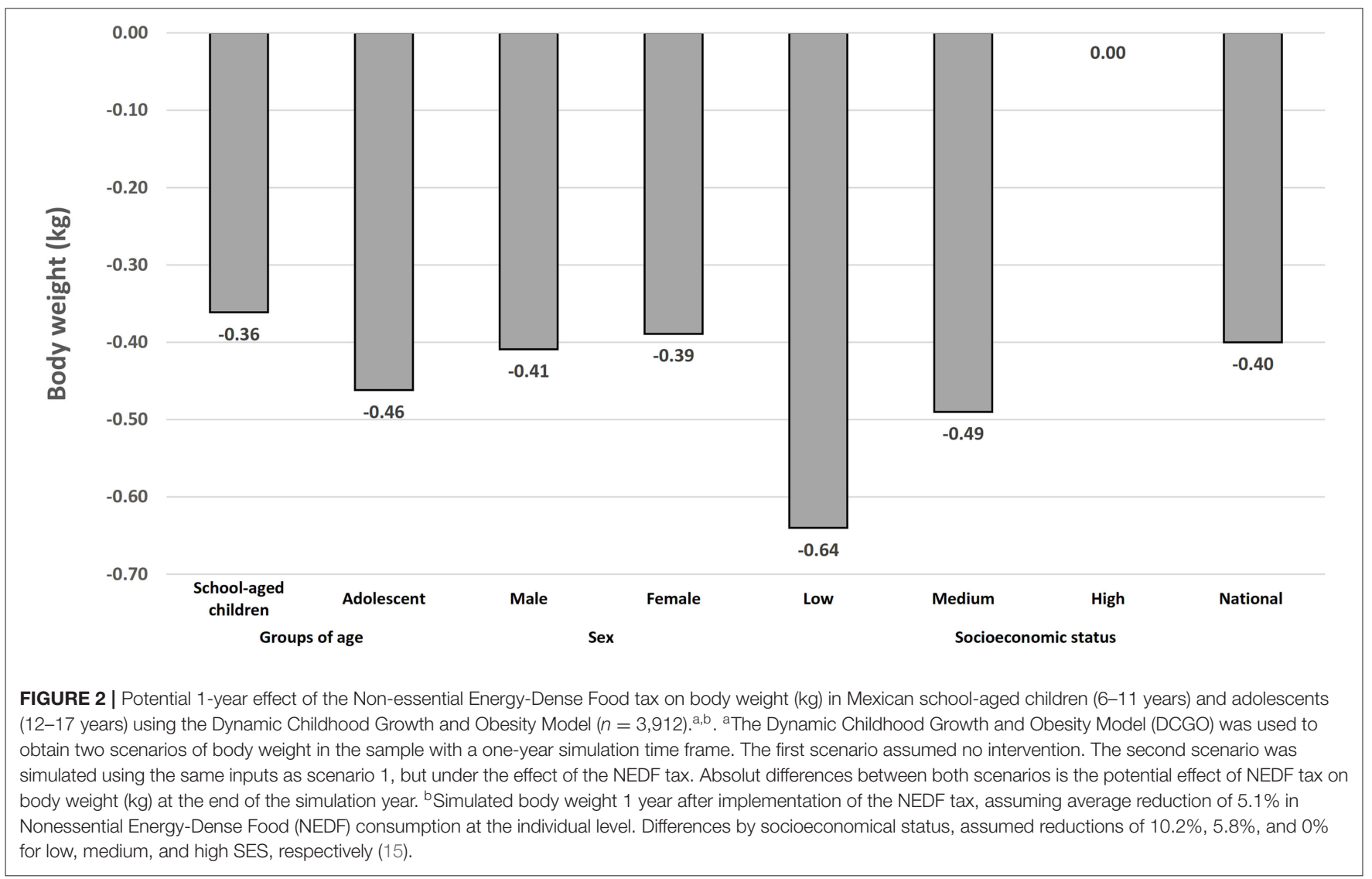

international comparison using the tax-based NEDF definition is difficult, but those NEDF are a subgroup of ultraprocessed foods according to the NOVA classification. In Mexico, schoolaged children consume $34.3 \%$ of their total energy intake from ultraprocessed foods, while adolescents consume $35.5 \%$ (31). In contrast, in 2005, Colombian children aged 2-9 years consume $18.5 \%$ of total energy intake, while adolescents consume $18.6 \%$ (32); in 2010, Chilean children 2-19 years consumed $37.6 \%$ of their total energy intake from ultraprocessed foods (33). In contrast, in Canada and in the US, ultraprocessed foods represent 
TABLE 3 | Potential impact 1 year after the implementation of the NEDF tax on overweight and obesity prevalence by sociodemographic characteristics using the Dynamic Childhood Growth and Obesity Model ${ }^{\mathrm{a}}$.

\begin{tabular}{|c|c|c|c|c|c|c|}
\hline & \multicolumn{3}{|c|}{ Overweight } & \multicolumn{3}{|l|}{ Obesity } \\
\hline & $\begin{array}{l}\text { Without tax } \\
{[\%(95 \% \mathrm{Cl})]}\end{array}$ & $\begin{array}{c}\text { With tax } \\
{[\%(95 \% \mathrm{CI})]}\end{array}$ & $\begin{array}{l}\text { Absolute difference } \\
\text { (pp) }\end{array}$ & $\begin{array}{l}\text { Without tax } \\
{[\%(95 \% \mathrm{Cl})]}\end{array}$ & $\begin{array}{c}\text { With tax } \\
{[\%(95 \% \mathrm{Cl})]}\end{array}$ & $\begin{array}{l}\text { Absolute difference } \\
\text { (pp) }\end{array}$ \\
\hline Total & $21.2(19,23)$ & $19.4(18,22)$ & $-1.7(-0.80,-2.5)$ & $13.8(12,15)$ & $13.4(11,15)$ & $-0.4(-0.10,-0.60)$ \\
\hline \multicolumn{7}{|l|}{ AGE GROUPS } \\
\hline School-aged children & $21.5(19,24)$ & $19.6(17,22)$ & $-1.8(-0.80,-2.7)$ & $13.6(12,16)$ & $13.1(11,15)$ & $-0.4(-0.10,-0.80)$ \\
\hline Adolescent & $20.7(18,24)$ & $19.1(16,22)$ & $-1.5(-0.10,-3.0)$ & $14.1(11,18)$ & $13.9(11,18)$ & $-0.2(-0.10,-0.40)$ \\
\hline \multicolumn{7}{|l|}{ SEX } \\
\hline Male & $20.6(18,24)$ & $18.7(16,22)$ & $-1.9(-0.50,-3.1)$ & $16.2(13,19)$ & $15.6(13,18)$ & $-0.5(-0.10,-0.90)$ \\
\hline Female & $21.7(19,25)$ & $20.2(17,23)$ & $-1.5(-0.40,-2.4)$ & $11.4(9.6,13)$ & $11.2(9,13)$ & $-0.2(-0.10,-0.40)$ \\
\hline \multicolumn{7}{|c|}{ SOCIOECONOMIC STATUS ${ }^{b}$} \\
\hline Low & $23.7(20,27)$ & $22.1(19,26)$ & $-1.9(-0.80,-2.9)$ & $11.3(9,14)$ & $10.8(9,13)$ & $-0.6(-0.01,-1.0)$ \\
\hline Medium & $20.3(17,24)$ & $17.7(15,21)$ & $-2.6(-0.60,-4.6)$ & $13.7(11,17)$ & $13.2(11,16)$ & $-0.5(-0.12,-0.96)$ \\
\hline High & $19.4(16,23)$ & $19.4(16,23)$ & $0.0(0.0,0.0)$ & $16.1(13,19)$ & $16.1(13,19)$ & $0.0(0.0,0.0)$ \\
\hline
\end{tabular}

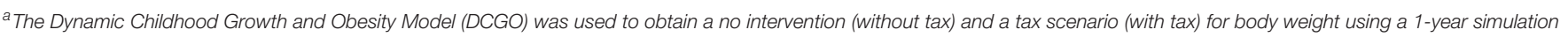
time frame; the absolute difference is the expected impact of the NEDF tax on overweight and obesity.

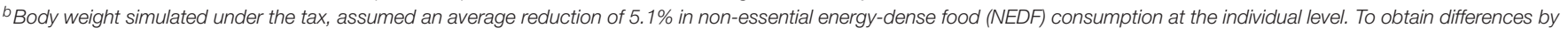
SES, we used the effects stratified as follows: 10.2, 5.8, and 0\% for low, medium, and high SES, respectively (15).

$B M I$, body mass index; NEDF, non-essential energy-dense foods; SE, standard error; pp, percentage points.

TABLE 4 | Sensitivity analyses for the potential 1-year effect of the NEDF tax on BMl and overweight and obesity, based on 10 or $20 \%$ energy intake compensation ( $n=$ 3,912).

\begin{tabular}{|c|c|c|c|c|}
\hline Simulated scenarios of the NEDF tax effect & BMI (mean \pm SE) & BMI for age $z$-score (mean \pm SE) & Overweight [pp $(95 \% \mathrm{Cl})]$ & Obesity [pp $(95 \% \mathrm{CI})]$ \\
\hline $\begin{array}{l}\text { Absolute difference }{ }^{a} \text { without energy } \\
\text { compensation }\end{array}$ & $-0.19 \pm 0.01$ & $-0.09 \pm 0.01$ & $-1.7(-0.80,-2.5)$ & $-0.4(-0.10,-0.60)$ \\
\hline $\begin{array}{l}\text { Dietary energy compensation } \\
\text { Absolute difference }{ }^{a} \text { with } 10 \% \text { of energy } \\
\text { compensation }\end{array}$ & $-0.17 \pm 0.01$ & $-0.08 \pm 0.01$ & $-1.4(-0.60,-2.1)$ & $-0.3(-0.10,-0.50)$ \\
\hline Absolute difference ${ }^{a}$ with $20 \%$ of energy & $-0.15 \pm 0.01$ & $-0.7 \pm 0.01$ & $-1.3(-0.60,-2.1)$ & $-0.3(-0.10,-0.50)$ \\
\hline
\end{tabular}

compensation

We assume that all the change in body weight occurs via reduction in NEDF consumption.

${ }^{a}$ Absolute difference between the no intervention scenario and the NEDF tax scenario. Difference calculated 1 year after the implementation of the tax.

BMI, body mass index; NEDF, non-essential energy-dense foods; SE, Standard error; pp, percentage points.

55 and $65 \%$ of the total energy intake, respectively (33-35). The tax in Mexico was observed to reduce NEDF consumption at least $5.1 \%$, which should lead to a reduction of $0.39 \mathrm{~kg} / \mathrm{m}^{2}$ in BMI and 0.09 fewer units in BMI-for-age $z$-score. This could seem small from an individual perspective, but at a population level, it is considerable. Moreover, results should be interpreted considering that we only modeled 1 year after implementation and that changes over time could extend beyond this period.

The NEDF tax represents an $8 \%$ price increase, which, compared to sugar-sweetened beverages taxes (10\%), is low and very low if compared to the $66.9 \%$ price increase produced by tobacco taxes in Mexico $(7,36)$. Tobacco taxes are an excellent example of the potential of taxes to reduce the use of harmful products; in tobacco control, adolescents have proven to be more responsive to taxes than adults, reducing $4 \%$ in cigarettes consumed for each $10 \%$ increase in price (36-38).

Achieving a reduction in the consumption of NEDF in children is an important objective to improve children's health. Lower NEDF consumption implies a significant improvement in diets, as NEDF are rich in added sugar, saturated fat, and sodium, which have been associated with obesity in children $(33-35,39,40)$. Body weight loss in children has been linked with improvement in blood pressure, low-density lipoprotein (LDL)-cholesterol, high-density lipoprotein (HDL)-cholesterol, triglycerides, or insulin resistance even if the BMI $z$-score reduction is small (41); thus, small but sustained weight loss or prevention of unhealthy weight gain could help reverse the burden of disease that derives from obesity in children.

To our knowledge, this is the first study to estimate the potential impact of the NEDF tax on body weight, BMI, and overweight and obesity prevalence in children. Consequently, we could not identify studies directly comparable to ours. However, to put our estimates into perspective, we will compare them with the effects achieved by randomized trials to reduce weight in children and with other simulation models. Empirical studies in children 2-18 years old, focusing on dietary improvement, have achieved reductions in BMI-for-age $z$-score of -0.04 (95\% CI: -0.08 to -0.01 ) (42) and up to -0.09 (95\% CI: -0.13 to 
$-0.05)$, which are within the range of our estimates as presented in Supplementary Table 1 (43). Our results are also within the range of effects estimated by previous modeling studies for other food policies in children. Brown et al., using the same mathematical model, estimated the potential effect of reducing 27 $\mathrm{kcal} /$ day associated to restrictions to TV advertising of beverages and foods in children 5-15 years of age, which was expected to reduce $0.35 \mathrm{~kg} / \mathrm{m}^{2}$ of BMI within 1 year (44); in contrast, we estimated a $0.19 \mathrm{~kg} / \mathrm{m}^{2}$ reduction from the $17.4 \mathrm{kcal} /$ day reduction expected from the NEDF tax. Torres-Alvarez et al. used the same model to estimate the impact of the sugar-sweetened beverages tax in Mexican children 5-17 years old; the 10\% tax is expected to reduce $17.6 \mathrm{kcal} /$ day, which should translate into a $0.42 \mathrm{~kg}$ body weight reduction (45).

The prevalence of overweight and obesity in school-aged children and adolescents is very high in Mexico (46). We estimated that the national prevalence of overweight and obesity effect should be higher in school-aged children, male children, and children in the low SES group. School-aged and male children are the groups with the highest NEDF intake among all children, which explains why the tax could produce larger decreases in these groups (14). In contrast, the larger reduction in body weight observed in low SES is related to a stronger response of this group to the NEDF tax, as observed by Batis et al. (15). While the estimated reduction in overweight and obesity is small, the prevention of obesity and overweight cases in this age group could lead to large improvements in cardiometabolic health later in life, considering that childhood obesity is a strong predictor of obesity and chronic diseases $(47,48)$. This is an important finding that points to the equitability of the NEDF tax, as children in the lowest quintile of SES in Mexico are experiencing the highest increases in overweight and obesity and will carry the heaviest burden of chronic diseases in the future (46). In that sense, the NEDF tax is a progressive measure, as it will likely reduce future healthcare costs for the less favored individuals (7). Given our estimates, it is evident that increases to the NEDF tax will be needed to produce larger reductions in the prevalence and that fiscal measures will need to be accompanied by other population strategies to help reduce overweight and obesity in children.

In ENSANUT 2012, it was observed that the mean energy consumption exceeds the total energy requirement by $10 \%$ in school-aged children and up to $7 \%$ in adolescents (23). This translates into an average imbalance of 190 and 165 extra $\mathrm{kcal} /$ day, respectively $(17,49)$. In the present study, energy reduction due to NEDF tax was estimated at $16.7 \mathrm{kcal}$ in schoolaged children and $18.5 \mathrm{kcal}$ in adolescents, which provides a quantitative estimate of the magnitude of the interventions needed to prevent obesity in future generations. Sugar-sweetened beverages tax is another intervention in Mexico designed to reduce energy consumption in the population, and the potential effect estimated was $11.7 \mathrm{kcal} /$ day in school-aged children and $24.4 \mathrm{kcal} /$ day in adolescents. These two interventions encompass just 15.0 and $26.0 \%$ of the excess in energy consumption in these groups of age. Therefore, none of these two interventions by itself would be sufficient to reverse the obesity trend in Mexico. Instead, a comprehensive strategy including effective interventions covering from pregnancy to adulthood to prevent excessive weight gain is needed (50). For instance, Australia has proposed a national strategy to reduce the obesity prevalence by increasing breastfeeding and physical activity, improving the food environment, and reducing the consumption of unhealthy foods through taxes, while increasing the consumption of healthy foods through subsidies (51).

Our study has some limitations that must be considered. First, the expected change in NEDF intake due to the tax was obtained from an observational study that estimated the impact of the tax in NEDF purchases (15); thus, we had to assume that the observed changes in household purchases translate into changes in individual-level intake. This assumption is common to prior studies that have estimated the impact of sugarsweetened beverages on body weight (52-55). We used the 5.1\% decrease in NEDF household purchases reported by Batis et al. as a conservative estimate, although a subsequent evaluation observed a mean reduction in NEDF purchases of $6.0 \%$ in $2014-$ 2015 , which is related to a larger reduction in purchases in the second year of tax (27). Therefore, the estimated effect on body weight and the prevalence of overweight and obesity in our study could be underestimated. Second, the model assumes a steady state, that is, it assumes that obesity rates are not increasing and that no other intervention affects the outcome. Third, we assume food compensation of 10 and $20 \%$ for a reduction in NEDF consumption, which implies that at least $80 \%$ of the effect of the intervention translates into a child's total energy expenditure and, subsequently, to body weight. There is no consensus on the compensatory effect for dietary energy reduction, especially in the long term (56). However, a recent study reduced $8 \%$ of energy density in preschoolers' diet (72 kcal/day) and maintained the gap without energy compensation during the trial (5 days) (57). This suggests that the reduction in NEDF consumption given the implementation of tax might have a similar effect to this study, in which energy reduction is maintained over time.

In conclusion, our study suggests that the NEDF tax should reduce overweight and obesity in children 5-17 years old. Our analysis by SES demonstrates a greater impact on those living in socioeconomic disadvantage compared to least disadvantaged children. The use of fiscal instruments to discourage consumption of NEDF is a strategy to tackle childhood obesity that should be considered an integral part of any overarching strategy to reduce childhood obesity. The current $8 \%$ tax should be increased to produce larger health gains.

\section{DATA AVAILABILITY STATEMENT}

The model developed by Hall et al. (58) and used for this study is available in $\mathrm{R}$ (http://github.com/INSP- RH/bw-Mexicanchildren-population). Data from ENSANUT 2012 used as inputs for the model are publicly available in www.ensanut.insp.mx $(16,17)$.

\section{ETHICS STATEMENT}

A signed assent form and signed parent/guardians consent form were required to obtain children's measurements and participate 
in ENSANUT 2012. This study was reviewed and approved by the ethics and research committees of the National Institute of Public Health of Mexico.

\section{AUTHOR CONTRIBUTIONS}

DI-Z and TB-G designed the study. RT-Á contributed to the model development and conducted the model simulations. DI-Z wrote the first draft of the manuscript and analyzed the data. All authors review model parameters, inputs, and provided important intellectual contributions to the revision of the manuscript and approval of the final manuscript.

\section{REFERENCES}

1. Butte NF, Christiansen E, Sørensen TI. Energy imbalance underlying the development of childhood obesity. Obesity. (2007) 15:3056-66. doi: 10.1038/oby.2007.364

2. Lin BH, Smith TA, Lee JY, Hall KD. Measuring weight outcomes for obesity intervention strategies: the case of a sugar-sweetened beverage tax. Econ Hum Biol. (2011) 9:329-41. doi: 10.1016/j.ehb.2011.08.007

3. Romieu I, Dossus L, Barquera S, Blottière HM, Franks PW, Gunter M, et al. Energy balance and obesity: what are the main drivers? Cancer Causes Control. (2017) 28:247-58. doi: 10.1007/s10552-017-0869-z

4. Di Cesare M, Sorić M, Bovet P, Miranda JJ, Bhutta Z, Stevens GA, et al. The epidemiological burden of obesity in childhood: a worldwide epidemic requiring urgent action. BMC Med. (2019) 17:212. doi: 10.1186/s12916-019-1449-8

5. INEGI. Encuesta Nacional de Salud y Nutrición 2018. ENSANUT. Diseño conceptual. (2019). Available online at: https://ensanut.insp.mx/index.php (accessed November 25, 2020).

6. Ng M, Fleming T, Robinson M, Thomson B, Graetz N, Margono C, et al. Global, regional, and national prevalence of overweight and obesity in children and adults during 1980-2013: a systematic analysis for the Global Burden of Disease Study 2013. Lancet. (2014) 384:766-81. doi: 10.1016/S0140-6736(14)60460-8

7. Barrientos-Gutierrez T, Colchero MA, Sanchez-Romero LM, Batis C, Rivera-Dommarco J. Position paper on taxes to non-basic energy-dense foods and sugar-sweetened beverages. Salud Públ Méx. (2018) 60:586-91. doi: $10.21149 / 9534$

8. Wang YC, McPherson K, Marsh T, Gortmaker SL, Brown M. Health and economic burden of the projected obesity trends in the USA and the UK. Lancet. (2011) 378(9793):815-25. doi: 10.1016/S0140-6736(11)60814-3

9. Drewnowski A, Specter SE. Poverty and obesity: the role of energy density and energy costs. Am J Clin Nutr. (2004) 79:6-16. doi: 10.1093/ajcn/79.1.6

10. Rouhani MH, Haghighatdoost F, Surkan PJ, Azadbakht L. Associations between dietary energy density and obesity: a systematic review and meta-analysis of observational studies. Nutrition. (2016) 32:1037-47. doi: 10.1016/j.nut.2016.03.017

11. Shroff MR, Perng W, Baylin A, Mora-Plazas M, Marin C, Villamor E. Adherence to a snacking dietary pattern and soda intake are related to the development of adiposity: a prospective study in school-age children. Public Health Nutr. (2014) 17:1507-13. doi: 10.1017/S136898001300133X

12. Mytton OT, Eyles H, Ogilvie D. Evaluating the health impacts of food and beverage taxes. Curr Obes Rep. (2014) 3:432-9. doi: 10.1007/s13679-014-0123-x

13. Congreso de los Estados Unidos Mexicanos. Ley del Impuesto Especial sobre Producción y Servicios Mexico (2014). Available online at: http:// www.diputados.gob.mx/LeyesBiblio/pdf/78_241219.pdf (accessed November 25, 2020).

14. Batis C, Pedraza LS, Sánchez-Pimienta TG, Aburto TC, Rivera-Dommarco JA. Energy, added sugar, and saturated fat contributions of taxed beverages and foods in Mexico. Salud Públ Méx. (2017) 59:512-7. doi: 10.21149/8517

\section{FUNDING}

This work was supported by a grant from Bloomberg Philanthropies (https://www.bloomberg.org/) to the National Institute of Public Health (PI JR).

\section{SUPPLEMENTARY MATERIAL}

The Supplementary Material for this article can be found online at: https://www.frontiersin.org/articles/10.3389/fpubh. 2020.591696/full\#supplementary-material

15. Batis C, Rivera JA, Popkin BM, Taillie LS. First-year evaluation of Mexico's tax on nonessential energy-dense foods: an observational study. PLoS Med. (2016) 13:e1002057. doi: 10.1371/journal.pmed.1002057

16. Gutiérrez JP, Rivera-Dommarco J, Shamah-Levy T, Villalpando-Hernández S, Franco A, Cuevas-Nasu L, et al. Encuesta Nacional de Salud y Nutrición 2012. Resultados Nacionales Cuernavaca, México: Instituto Nacional de Salud Pública (2012). Available online at: https://ensanut.insp.mx/ (accessed November 25, 2020).

17. Hall KD, Butte NF, Swinburn BA, Chow CC. Dynamics of childhood growth and obesity: development and validation of a quantitative mathematical model. Lancet Diabetes Endocrinol. (2013) 1:97-105. doi: 10.1016/S2213-8587(13)70051-2

18. De Onis M. WHO Child Growth Standards: Methods and DevelopmentLength/Height-for-Age, Weight-for-Age, Weight-for-Length, Weight-for-Height and Body Mass Index-for-Age. (2006). Available online at: https://www.who. int/childgrowth/standards/Technical_report.pdf?ua=1 (accessed November 25, 2020).

19. Romero-Martínez M, Shamah-Levy T, Franco-Núñez A, Villalpando S, Cuevas-Nasu L, Gutiérrez JP, et al. Encuesta Nacional de Salud y Nutrición 2012: diseño y cobertura. Salud Publ Mex. (2013) 55:S332-40. Available online at: http://www.scielo.org.mx/pdf/spm/v55s2/v55s2a33.pdf (accessed November 25, 2020).

20. Habicht JP. Estandarización de métodos epidemiológicos cuantitativos sobre el terreno. PAHO Bull. (1974) 76:375-84. Available from: https://iris.paho. $\mathrm{org} / \mathrm{bitstream} /$ handle/10665.2/766/v76n5p375.pdf?sequence $=1 \quad$ (accessed November 25, 2020).

21. Lohman T, Roche A, Martorell R. Anthropometric Standardization Reference Manual Abridged Edition: Human Kinetics Books. (1991). Available online at: http://books.google.com.mx/books?id=wgd9QgAACAAJ (accessed November 25, 2020).

22. World Health Organization. WHO AnthroPlus for Personal Computers Manual: Software for Assessing Growth of the World's Children and Adolescents. Geneva: WHO (2009). Available from: https://www.who.int/growthref/tools/ en/ (accessed November 25, 2020).

23. López-Olmedo N, Carriquiry AL, Rodríguez-Ramírez S, Ramírez-Silva I, Espinosa-Montero J, Hernández-Barrera L, et al. Usual intake of added sugars and saturated fats is high while dietary fiber is low in the Mexican population. J Nutr. (2016) 146:1856S-65S. doi: 10.3945/jn.115.218214

24. Conway JM, Ingwersen LA, Moshfegh AJ. Accuracy of dietary recall using the USDA five-step multiple-pass method in men: an observational validation study. J Am Diet Assoc. (2004) 104:595-603. doi: 10.1016/j.jada.2004.01.007

25. Ramírez-Silva I, Barragán-Vázquez S, Mongue-Urea A, Mejía-Rodríguez F, Rodríguez-Ramírez S, Martínez-Piña A, et al. Base de Alimentos de México 2012 (BAM): Compilación de la composición de los alimentos frecuentemente consumidos en el país. Instituto Nacional de Salud Pública (2020). Version 18.1.2. Available online at: http://kin.insp.mx/aplicaciones/ Redpidiet (accessed December 28, 2020).

26. Gutiérrez JP. Clasificación socioeconómica de los hogares en la ENSANUT 2012. Salud Públ Méx. (2013) 55:S341-S6. Available online at: http://www. scielo.org.mx/pdf/spm/v55s2/v55s2a34.pdf (accessed November 25, 2020). 
27. Taillie LS, Rivera JA, Popkin BM, Batis C. Do high vs. low purchasers respond differently to a nonessential energy-dense food tax? Two-year evaluation of Mexico's 8\% nonessential food tax. Prev Med. (2017) 105:S37-42. doi: 10.1016/j.ypmed.2017.07.009

28. Hernández-F M, Batis C, Rivera JA, Colchero MA. Reduction in purchases of energy-dense nutrient-poor foods in Mexico associated with the introduction of a tax in 2014. Prev Med. (2019) 118:16-22. doi: 10.1016/j.ypmed.2018.09.019

29. StataCorp L. Stata 13. College Station: StataCorp LP. (2014). Available online at: https://www.stata.com/company/ (accessed November 25, 2020).

30. R Core Team. R: Language and Environment for Statistical Computing. $R$ Foundation for Statistical Computing. Vienna, Austria: R Core Team (2019).

31. Marrón-Ponce JA, Sánchez-Pimienta TG, Louzada M, Batis C. Energy contribution of NOVA food groups and sociodemographic determinants of ultra-processed food consumption in the Mexican population. Public Health Nutr. (2018) 21:87-93. doi: 10.1017/s1368980017002129

32. Khandpur N, Cediel G, Obando DA, Jaime PC, Parra DC. Sociodemographic factors associated with the consumption of ultra-processed foods in Colombia. Rev Saúde Pública. (2020) 54:19. doi: 10.11606/s1518-8787.2020054001176

33. Cediel G, Reyes M, da Costa Louzada ML, Steele EM, Monteiro CA, Corvalán C, et al. Ultra-processed foods and added sugars in the Chilean diet. (2010). Public Health Nutr. (2018) 21:125-33. doi: 10.1017/S1368980017001161

34. Moubarac J-C, Batal M, Louzada M, Steele EM, Monteiro C. Consumption of ultra-processed foods predicts diet quality in Canada. Appetite. (2017) 108:512-20. doi: 10.1016/j.appet.2016.11.006

35. Neri D, Martinez-Steele E, Monteiro CA, Levy RB. Consumption of ultraprocessed foods and its association with added sugar content in the diets of US children, NHANES 2009-2014. Pediatr Obes. (2019) 14:e12563. doi: 10.1111/ijpo.12563

36. Reynales-Shigematsu LM, Wipfli H, Samet J, Regalado-Pineda J, HernándezÁvila M. Tobacco control in Mexico: a decade of progress and challenges. Salud Públ Méx. (2020) 61:292-302. doi: 10.21149/9360

37. Ding A. Youth are more sensitive to price changes in cigarettes than adults. Yale J Biol Med. (2003) 76:115-24.

38. Guindon GE, Paraje GR, Chaloupka FJ. Association of tobacco control policies with youth smoking onset in Chile. JAMA Pediatr. (2019) 173:754-62. doi: 10.1001/jamapediatrics.2019.1500

39. Khandpur N, Neri DA, Monteiro C, Mazur A, Frelut M-L, Boyland E, et al. Ultra-processed food consumption among the paediatric population: an overview and call to action from the European Childhood Obesity Group. Ann Nutr Metab. (2020) 76:109-13. doi: 10.1159/000 507840

40. Sánchez-Pimienta TG, Batis C, Lutter CK, Rivera JA. Sugar-sweetened beverages are the main sources of added sugar intake in the Mexican population. J Nutr. (2016) 146:1888S-96S. doi: 10.3945/jn.115.220301

41. Reinehr T, Lass N, Toschke C, Rothermel J, Lanzinger S, Holl RW. Which amount of BMI-SDS reduction is necessary to improve cardiovascular risk factors in overweight children? J Clin Endocrinol Metab. (2016) 101:3171-9. doi: 10.1210/jc.2016-1885

42. Sim LA, Lebow J, Wang Z, Koball A, Murad MH. Brief primary care obesity interventions: a meta-analysis. Pediatrics. (2016) 138:e20160149. doi: 10.1542/peds.2016-0149

43. Taveras EM, Marshall R, Sharifi M, Avalon E, Fiechtner L, Horan C, et al. Comparative effectiveness of clinical-community childhood obesity interventions: a randomized clinical trial. JAMA Pediatr. (2017) 171:e171325e. doi: 10.1001/jamapediatrics.2017.1325

44. Brown V, Ananthapavan J, Veerman L, Sacks G, Lal A, Peeters A, et al. The potential cost-effectiveness and equity impacts of restricting television advertising of unhealthy food and beverages to Australian children. Nutrients. (2018) 10:622. doi: 10.3390/nu10050622

45. Torres-Álvarez R, Barrán-Zubaran R, Canto-Osorio F, Sánchez-Romero L, Camacho-García-Formentí D, Popkin B, et al. Body weight impact of the sugar-sweetened beverages tax in Mexican children: a modeling study. Pediatr Obes. (2020) 15:1-8. doi: 10.1111/ijpo.12636

46. Hernández-Cordero S, Cuevas-Nasu L, Morales-Ruán M, Humarán IM-G, Ávila-Arcos M, Rivera-Dommarco J. Overweight and obesity in Mexican children and adolescents during the last 25 years. Nutr Diabetes. (2017) 7:e247. doi: 10.1038/nutd.2016.52

47. Umer A, Kelley GA, Cottrell LE, Giacobbi P, Innes KE, Lilly CL. Childhood obesity and adult cardiovascular disease risk factors: a systematic review with meta-analysis. BMC Publ Health. (2017) 17:683. doi: 10.1186/s12889-017-4691-z

48. Whitaker RC, Wright JA, Pepe MS, Seidel KD, Dietz WH. Predicting obesity in young adulthood from childhood and parental obesity. N Engl J Med. (1997) 337:869-73. doi: 10.1056/NEJM199709253371301

49. Wang YC, Orleans CT, Gortmaker SL. Reaching the healthy people goals for reducing childhood obesity: closing the energy gap. Am J Prev Med. (2012) 42:437-44. doi: 10.1016/j.amepre.2012.01.018

50. Gortmaker SL, Long MW, Resch SC, Ward ZJ, Cradock AL, Barrett JL, et al. Cost Effectiveness of Childhood Obesity Interventions: Evidence and Methods for CHOICES. Am J Prev Med. (2015) 49:102-11. doi: 10.1016/j.amepre.2015.03.032

51. Roberts N, Li V, Atkinson JA, Heffernan M, McDonnell G, Prodan A, et al. Can the target set for reducing childhood overweight and obesity be met? A System Dynamics Modelling Study in New South Wales, Australia. Syst Res Behav Sci. (2019) 36:36-52. doi: 10.1002/sres.2555

52. Barrientos-Gutierrez T, Zepeda-Tello R, Rodrigues ER, Colchero-Aragones A, Rojas-Martinez R, Lazcano-Ponce E, et al. Expected population weight and diabetes impact of the 1-peso-per-litre tax to sugar sweetened beverages in Mexico. PLoS ONE. (2017) 12:e0176336. doi: 10.1371/journal.pone.0176336

53. Basto-Abreu A, Torres-Alvarez R, Reyes-Sánchez F, González-Morales R, Canto-Osorio F, Colchero MA, et al. Predicting obesity reduction after implementing warning labels in Mexico: a modeling study. PLoS Med. (2020) 17:e1003221. doi: 10.1371/journal.pmed.1003221

54. Colchero MA, Rivera-Dommarco J, Popkin BM, Ng SW. In Mexico, evidence of sustained consumer response two years after implementing a sugar-sweetened beverage tax. Health Aff. (2017) 36:564-71. doi: $10.1377 /$ hlthaff.2016.1231

55. Sánchez-Romero LM, Penko J, Coxson PG, Fernández A, Mason A, Moran AE, et al. Projected impact of Mexico's sugar-sweetened beverage tax policy on diabetes and cardiovascular disease: a modeling study. PLoS Med. (2016) 13:e1002158. doi: 10.1371/journal.pmed.1002158

56. Almiron-Roig E, Palla L, Guest K, Ricchiuti C, Vint N, Jebb SA, et al. Factors that determine energy compensation: a systematic review of preload studies. Nutr Rev. (2013) 71:458-73. doi: 10.1111/nure.12048

57. Smethers AD, Roe LS, Sanchez CE, Zuraikat FM, Keller KL, Rolls BJ. Both increases and decreases in energy density lead to sustained changes in preschool children's energy intake over 5 days. Physiol Behav. (2019) 204:210-8. doi: 10.1016/j.physbeh.2019. 02.042

58. Camacho-Garcia-Fomenti D, Zepeda-Tello R. BW: Dynamic Body Weight Models for Children and Adults. R Package (2018). (version 1.0.1). Mexico.

Conflict of Interest: The authors declare that the research was conducted in the absence of any commercial or financial relationships that could be construed as a potential conflict of interest.

Copyright (c) 2021 Illescas-Zárate, Batis, Ramírez-Silva, Torres-Álvarez, Rivera and Barrientos-Gutiérrez. This is an open-access article distributed under the terms of the Creative Commons Attribution License (CC BY). The use, distribution or reproduction in other forums is permitted, provided the original author $(s)$ and the copyright owner(s) are credited and that the original publication in this journal is cited, in accordance with accepted academic practice. No use, distribution or reproduction is permitted which does not comply with these terms. 